\title{
Optimizing the achievable rates of tricky channels: a probabilistic shaping for OPC channel example
}

Yankov, Metodi Plamenov; Da Ros, Francesco; Porto da Silva, Edson; Forchhammer, Søren; Galili, Michael; Oxenløwe, Leif Katsuo

Published in:

Proceedings of 31st Annual Conference of the IEEE Photonics Society

Publication date:

2018

Document Version

Peer reviewed version

Link back to DTU Orbit

Citation (APA):

Yankov, M. P., Da Ros, F., Porto da Silva, E., Forchhammer, S., Galili, M., \& Oxenløwe, L. K. (2018). Optimizing the achievable rates of tricky channels: a probabilistic shaping for OPC channel example. In Proceedings of 31 st Annual Conference of the IEEE Photonics Society IEEE.

\section{General rights}

Copyright and moral rights for the publications made accessible in the public portal are retained by the authors and/or other copyright owners and it is a condition of accessing publications that users recognise and abide by the legal requirements associated with these rights.

- Users may download and print one copy of any publication from the public portal for the purpose of private study or research.

- You may not further distribute the material or use it for any profit-making activity or commercial gain

- You may freely distribute the URL identifying the publication in the public portal 


\section{Optimizing the achievable rates of tricky channels: a probabilistic shaping for OPC channel example}

(Invited paper)

\author{
Metodi P. Yankov ${ }^{1,2}$ \\ ${ }^{1}$ Fingerprint Cards $\mathrm{A} / \mathrm{S}$ \\ 2730 Herlev, Denmark \\ meya@fotonik.dtu.dk \\ metodi.yankov@fingerprints.com
}

\author{
Francesco Da Ros ${ }^{1}$, Edson Porto da Silva ${ }^{1}$, Søren Forchhammer ${ }^{1}$, \\ Michael Galili ${ }^{1}$, and Leif K. Oxenløwe ${ }^{1}$ \\ ${ }^{2}$ Department of Photonics Engineering \\ Technical University of Denmark \\ 2800 Kgs. Lyngby, Denmark
}

\begin{abstract}
A method is presented for online probabilistic shaping parameter optimization for channels, which are nontrivial to model and are thus difficult to optimize offline. An example is provided for a mid-link optical phase conjugation based nonlinearity compensation channel with inline dispersion compensation.
\end{abstract}

Index Terms-Probabilistic shaping, OPC, optimization

\section{INTRODUCTION}

In recent years, probabilistic shaping (PS) has become a more and more popular method for improving the achievable information rates (AIRs) of optical communication channels and bringing them closer to capacity. Standard approaches for shaping rely on transmitting e.g. a quadrature amplitude modulation (QAM) signal with a probability mass function (PMF), originating in the Maxwell-Boltzmann family [1]. While MB PMFs are near-optimal on additive white Gaussian noise (AWGN) channels, the nonlinearities in fibers make the channel non-trivial to optimize, with several approaches investigated in the literature [2]. Furthermore, offline, simulationbased PMF optimization can neglect some important properties of the channel due to the difficulty in modelling all hardware impairments, present in real-life systems.

In this paper, an optimization method is presented for the signal PMF which employs the physical channel, and thus includes all imperfections present in the system - components, digital signal processing (DSP), fiber, etc. - and more importantly, their true statistics. The methodology is demonstrated via a link, whose exact channel model is difficult to define, particularly - a mid-link optical phase conjugation (ML-OPC)based nonlinearity compensated channel.

\section{Probabilistic Optimization}

Optimization of the PMF of the input QAM signal is performed with the modified Blahut-Arimoto (BA) algorithm as in [3] and is briefly explained in Fig. 1. The BA algorithm (BAA) utilizes the expectation-maximization (E-M) algorithm

This work is supported by DNRF research Centre of Excellence SPOC, ref DNRF123

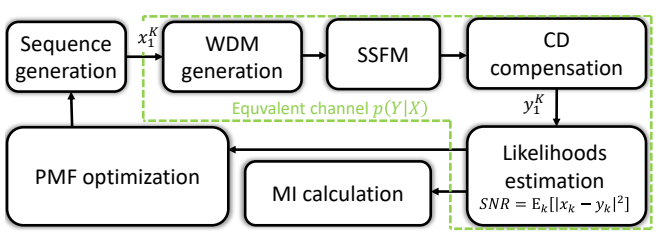

Fig. 1. Basic building blocks of the modified BAA [3].

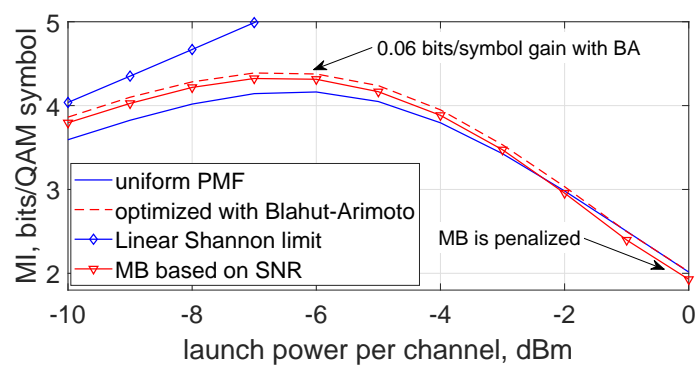

Fig. 2. AIRs with the algorithm from Fig. 1, together with AIRs with a PMF, optimized on an AWGN channel with equivalent SNR to the SNR, estimated on the SSFM channel.

to iteratively optimize a cost function (in this case the mutual information (MI)). On each iteration, a signal with the current PMF is propagated through the fiber (modelled by the spiltstep Fourier method (SSFM)), its statistics are extracted at the receiver, and used to generate a new PMF, which achieves higher MI. On the fiber channel, the BAA is only approximate and MI convergence is not guaranteed since the channel itself is highly dependent on the input PMF [4]. However, some of this dependency can be captured. This is shown in Fig. 2, where the MI with the BAA is shown, together with the MI, achieved with a Maxwell-Boltzmann (MB) PMF, near-optimal for on AWGN channel with equivalent signal to noise ratio (SNR). The BAA achieves around 0.06 bits/symbol gain over the basic MB shaping. In the high launch power regime, the SNR-based MB PMF is even penalized w.r.t. non-shaped PMF since it doesn't account for the statistics of the nonlinearities beyond the 2 nd order, i.e. the SNR.

\section{OPC-BASED NONLINEARITY COMPENSATION}

The experimental setup is shown in Fig. 3 [5]. The transmitter consist of 5 external cavity lasers (ECL, 10-kHz linewidth) 


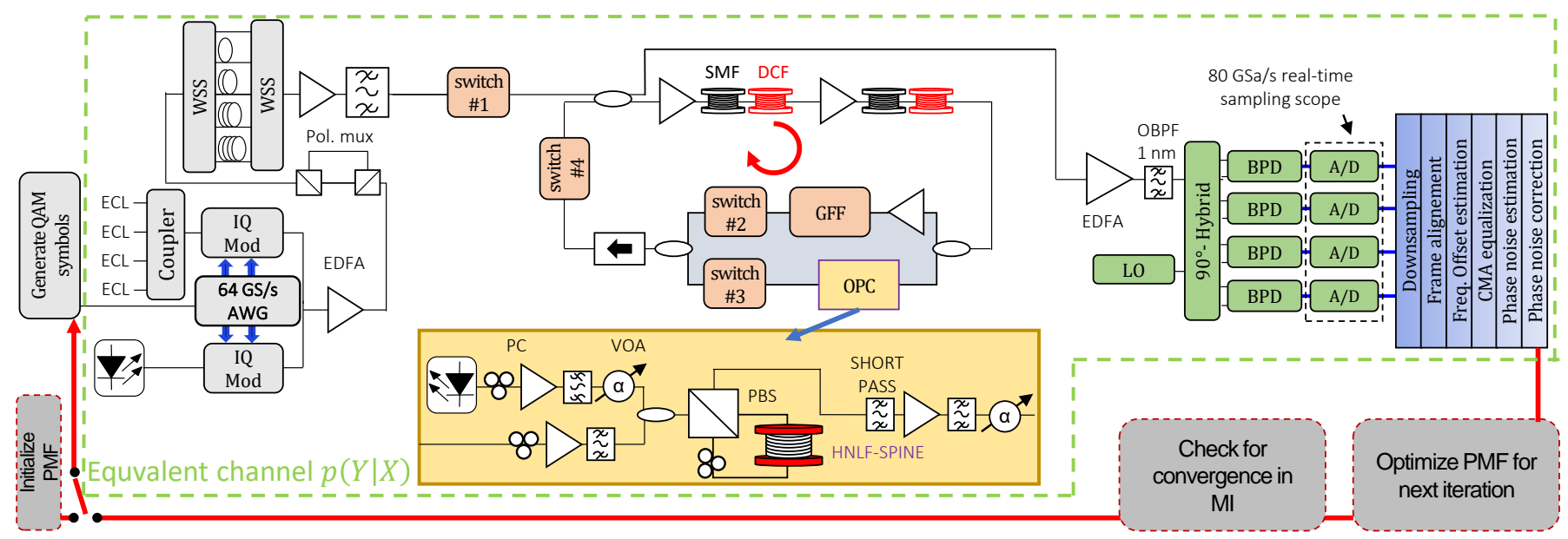

Fig. 3. Experimental setup. The OPC stage is described in details in [6]. The equivalent channel includes all hardware and software blocks between the symbol generation and the received sequence, used for MI estimation.

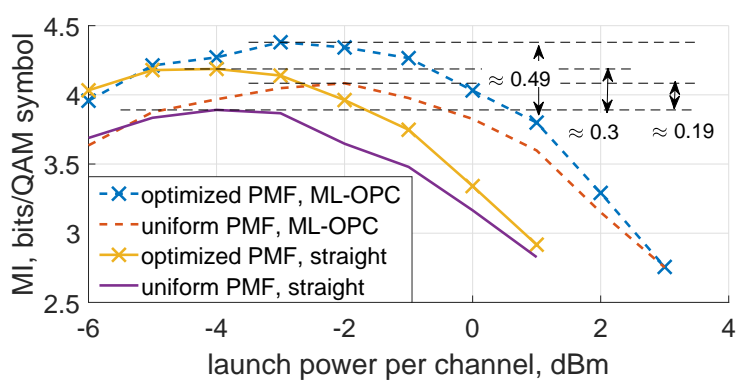

Fig. 4. AIR for straight and ML-OPC transmission with 64QAM. The gains from PS and ML-OPC appear to be independent, with those from PS being slightly higher.

modulated at $16 \mathrm{GBd}$ by two IQ modulators (one for the central channel under test and one for the four interfering channels) driven by an arbitrary waveform generator (AWG, 64 Gsamples/s, $20 \mathrm{GHz}$ bandwidth). The channels are then combined, delay-and-add polarization emulated and decorrelated using delay lines and wavelength selective switches (WSSs). The WDM channels are then launched into a recirculating transmission loop composed of two dispersion-compensated spans $(90$ and $50 \mathrm{~km}$, respectively, including the dispersion compensation spans), for a total loop length of $140 \mathrm{~km}$. Inside the loop, opto-acustic switches select either the straight path consisting of an EDFA and a gain flattening filter (GFF) (switch \#2), or the OPC path (switch \#3). The OPC is based on single-pump four-wave mixing stage in a highly nonlinear fiber (HNLF) in a polarization-diversity loop configuration [6]. At the output of the OPC, only the idler band is selected for further propagation. After transmission, the signal is received with a pre-amplified coherent receiver based on a 10-kHz local oscillator (LO) and a digital storage oscilloscope (DSO, 80$\mathrm{GSa} / \mathrm{s}$ and $33-\mathrm{GHz}$ analog bandwidth) performing the analogto-digital conversion. Pilot-based digital signal processing is performed as in [3].

\section{RESUlts}

Optimization of the PMF is performed for the system in Fig. 3 by plugging its equivalent channel (all hardware and software within the green dashed lines) in the BA framework
(Fig 1). The optimization iterations, AWG loading, reading received samples from the DSO and DSP for each iteration are performed "online" and within the same software instance. The MI, which also represents an AIR of the respective channel as a function of the launch power is given in Fig. 4 for straight (solid lines) and ML-OPC (dashed lines) transmission. The two techniques achieve gain through different means - MLOPC aims at reducing the nonlinear interference noise (NLIN) variance, while PS aims at optimizing the transmission for a given NLIN. The optimal launch power with ML-OPC is thus increased, while with PS, the system is pushed to operate at a lower launch power region, for which the noise is more Gaussian and independent, identically distributed in time. For the considered setup, ML-OPC provides $\approx 0.19$ bits/symbol of gain, and PS $\approx 0.3$ for both cases of straight and ML-OPC transmission. Furthermore, the gains of both techniques appear to add-up. We therefore conclude that the two techniques are independent to the extent of this analysis, and that even though the optimal launch power is different, the NLIN statistics of ML-OPC links and standard links at the optimal launch power, are qualitatively similar.

\section{CONClusion}

An online optimization methodology was presented, which allows for the optimization of transmitter parameters, particularly, the input probability mass function (PMF) for the physical channel under test. The methodology is "plug-and-play", and can be applied for any E-M type optimization problems. This is particularly important for links, which are difficult to model and/or have unknown properties and statistics, including unknown digital/optical transceiver, channel and digital signal processing imperfections.

\section{REFERENCES}

[1] T. Fehenberger et.al., JLT, 34(21), 2016

[2] J. Renner et.al., JLT, 35(22), 2017

[3] M. P. Yankov et.al., JLT, 34(22), 2016

[4] R. Dar et.al., OptExp, 21(22), 2013

[5] M. P. Yankov, et.al., CLEO, 2018, p. STu4C.2.

[6] F. Da Ros et.al., ECOC, 2017, p. P1.SC4.66. 